\title{
Significado do Programa Vivência e Estágios na Realidade do Sistema Único de Saúde para formação profissional na área de saúde*
}

\author{
Meaning of the Experience and Training Program in the Reality of the Single Health System for \\ professional bealth education
}

Significado del Programa Vivencia y Prácticas de enfermería en la Realidad del Sistema Único de Salud para la formación profesional en el área de salud

\author{
Rhavana Pilz Canônico ${ }^{1}$, Ana Cristina Passarella Brêtas ${ }^{2}$
}

\begin{abstract}
RESUMO
Objetivo: Este estudo qualitativo objetivou conhecer o significado do Programa Vivência e Estágios na Realidade do Sistema Único de Saúde (VER-SUS) para a formação do acadêmico da saúde. Métodos: Participaram dez graduandos de escolas públicas e privadas. Os dados foram analisados pela técnica da análise temática, na qual emergiram três categorias: o conhecimento do SUS no processo de formação; o VER-SUS como instrumento para inserção na política estudantil e/ou movimentos sociais; o VER-SUS e a formação acadêmica. Resultados: Os resultados revelaram que a participação no VER-SUS contribuiu para o aprimoramento individual, destacando sua importância, enquanto política pública. Foi um fator desencadeador da participação em movimentos sociais, particularmente no estudantil e apesar da qualidade da vivência, dificilmente será aplicada pelo coletivo, em decorrência de pouco conhecimento e/ou reduzido interesse dos docentes sobre o SUS. Conclusão: O VER-SUS é importante para a formação, apesar da pequena abrangência, pode contribuir para a formação política dos futuros profissionais. Possibilita a inserção interdisciplinar e interprofissional na formação.

Descritores: Sistema Único de Saúde; Saúde pública; Educação; Política de saúde; Recursos humanos em saúde; Capacitação de recursos humanos em saúde
\end{abstract}

\begin{abstract}
Objective: This qualitative study aimed to get to know the meaning of the Experience and Training Program in the Reality of the Single Health System (VER-SUS) for healthcare students' education. Methods: Participants were ten students from public and private schools. Semi-structured questions were used for data collection. The answers were subject to thematic analysis, revealing three categories: knowledge about SUS in the education process; VER-SUS as an instrument for insertion into academic policies and/or other social movements; VERSUS and academic education. Results: The results showed that participation in VER-SUS contributed to individual improvement, highlighting its importance as a public policy. It represented a triggering factor of participation in social and especially student movements. Despite the quality of the experience, application by the group will be difficult, due to the faculty members' lack of knowledge and/or little interest in SUS. Conclusion: VER-SUS is important for education, despite its small range, and can contribute to the political education of future professionals. It permits interdisciplinary and multiprofessional insertion into education.
\end{abstract}

Keywords: Single Health System; Public health; Education; Health policy; Health manpower; Health human resource training

\section{RESUMEN}

Objetivo: En este estudio cualitativo se tuvo como objetivo conocer el significado del Programa Vivencia y Prácticas de enfermería en la Realidad del Sistema Único de Salud (VER-SUS) para la formación del académico de la salud. Métodos: Participaron diez graduandos de escuelas públicas y privadas. Los datos fueron analizados por la técnica del análisis temática, en la cual emergieron tres categorías: el conocimiento del SUS en el proceso de formación; el VER-SUS como instrumento para la inserción en la política estudiantil y/o movimientos sociales; el VER-SUS y la formación académica. Resultados: Los resultados revelaron que la participación en el VER-SUS contribuyó en el pefeccionamiento individual, destacando su importancia, en cuanto a la política pública. Fue un factor desencadenante de la participación en movimientos sociales, particularmente en el estudiantil y a pesar de la calidad de la vivencia, dificilmente será aplicada por el colectivo, en consecuencia del poco conocimiento y/o reducido interés de los docentes sobre el SUS. Conclusión: El VER-SUS es importante para la formación, a pesar de lo poco que abarca, puede contribuir para la formación política de los futuros profesionales. Posibilita la inserción interdisciplinaria e interprofesional en la formación.

Descriptores: Sistema Único de Salud; Salud pública; Educación; Política de salud; Recursos humanos en salud; Capacitación de recursos humanos en salud

\footnotetext{
* Trabalho realizado nos encontros estudantis no Estado de São Paulo nos meses de marco e abril de 2005.

${ }^{1}$ Enfermeira do Programa "A gente na rua" da Secretaria Municipal de Saúde de São Paulo. São Paulo (SP), Brasil.

${ }^{2}$ Professora adjunto do Departamento de Enfermagem da Universidade Federal de São Paulo - UNIFESP - São Paulo (SP), Brasil.
} 


\section{INTRODUÇÃO}

O Sistema Único de Saúde (SUS) foi criado na Constituição de 1988 e regulamentado pela Lei Orgânica de Saúde (Lei no 8.080/90 e Lei 8142/90). A primeira Lei "regula, em todo o território Nacional, as ações e serviços de saúde, executados por pessoas naturais ou jurídicas de direito público ou privado". A segunda, que trata da participação da comunidade na gestão do SUS e das transferências inter-governamentais de recursos financeiros na área da saúde. Enquanto sistema busca garantir o acesso da população brasileira à saúde, visando minimizar as desigualdades de assistência de saúde no país. Defende que a saúde é um direito de todos e dever do Estado. Possui três princípios doutrinários: universalidade que determina a todos o acesso à saúde, sem discriminação de cor, etnia, classe social ou outras características pessoais ou sociais; eqüidade que objetiva diminuir desigualdades, tratando todos igualmente e; integralidade que proclama o ser humano como um todo e não em partes, tendo que ser atendido em todas as suas necessidades bio-psico-sociais em todos os níveis hierárquicos do sistema. Financeiramente o SUS é gerido pelos impostos e contribuições sociais pagos pela população e arrecadados pelos governos federal, estadual e municipal. Estes, por sua vez, têm o dever de redistribuir a quantia para suprir as necessidades locais de recursos materiais e humanos. Quando os recursos públicos são insuficientes, o SUS conta com a participação complementar do setor privado por meio de contratos e convênios ${ }^{(1)}$.

De acordo com a Resolução no 225/97 do Conselho Nacional de Saúde, o SUS tem o dever de contribuir com a formação de profissionais para a saúde, certamente um dos desafios do sistema e responsável em grande parte pelo êxito, ou não, na sua implementação( ${ }^{(2)}$.

O SUS necessita de profissionais preparados para atuar com competência na área da saúde pública, questão trabalhada nos serviços por meio do desenvolvimento de programas de educação continuada e reuniões técnico-científicas. Entretanto, ainda é visível na rede pública de saúde o despreparo técnico, científico e político de alguns trabalhadores ${ }^{(3)}$. Concomitantemente a essa constatação, aliase o fato das instituições de ensino superior (IES) que possuem cursos da área da saúde direcionarem o aprendizado mais no modelo biomédico do que nos paradigmas da Saúde Pública, ensinando de forma não comprometida com o sistema. Apesar de parte significativa dos conteúdos ser desenvolvida nos equipamentos de saúde do SUS, docentes e discentes usufruem do campo de prática, muitas vezes, sem dar nada em troca, ao contrário usam o sistema público para formar profissionais para o privado - caracterizando falta de compromisso ético com os usuários e com o SUS.

Neste contexto, em 2002 o Ministério da Saúde cria a Assessoria de Relações com o Movimento Estudantil e Associações Científico-Profissionais da Saúde, objetivando aproximar estudantes do desenvolvimento de projetos que visam estabelecer uma política de educação para futuros profissionais do SUS. Surgem diversas propostas de vivência, entre elas a Vivência e Estágios na Realidade do Sistema Único de Saúde (VER-SUS), que teve como versão piloto a vivência no Estado do Rio Grande do Sul, sendo posteriormente ampliada para outros estados brasileiros. Foi inserido na política de Educação para o Sistema Único de Saúde (EducarSUS) concebida pelo Ministério da Saúde com a finalidade de discutir e objetivar uma formação de qualidade para o SUS, capacitar profissionais de saúde, estimular a mudança curricular na graduação e especialização dos cursos da área da saúde, bem como a educação popular em saúde, utilizando práticas inovadoras de educação na área da saúde(2).

O VER-SUS tem como eixos principais propiciar oportunidade aos participantes para vivenciar conquistas e desafios inerentes ao SUS e aprofundar a discussão sobre o trabalho em equipe, gestão, atenção à saúde, educação e controle social. Propicia, também, discussões sobre a importância dos movimentos sociais, principalmente o movimento estudantil.

Historicamente, os movimentos sociais progressistas foram grandes articuladores no processo de construção do SUS, dentre eles se destaca o da Reforma Sanitária que no início da década de 80, lutou pela viabilização do ideário de um sistema de saúde público, com acesso universal e equânime a todos os brasileiros, fato consolidado em 1988 na Constituição Nacional. Assim, pode-se dizer que o SUS está instituído legalmente, mas a sua legitimação depende de um esforço concentrado da sociedade brasileira.

À guisa de buscar essa legitimidade o VER-SUS cumpre o seu papel enquanto política de educação para o SUS. Idealizado pelo movimento estudantil e concretizado pela parceria com o Ministério da Saúde objetiva, por meio da vivência de graduandos nos serviços públicos de saúde, estimularo senso crítico estudantil provocando reflexões acerca do papel do educando como transformador da realidade social; valorizar o compromisso dos participantes na implantação/implementação do SUS; contribuir para a construção do conceito ampliado de saúde; sensibilizar profissionais da área da saúde para fomentar discussões acerca do processo de educação permanente; contribuir para o amadurecimento da concepção interdisciplinar, intersetorial e interinstitucional; refletir sobre a política pedagógica nas instituições de ensino, visando a implementação do conhecimento aprofundado do SUS nas diretrizes curriculares; e estimular a inserção de estudantes no movimento estudantil e em outros movimentos sociais progressistas. Sem dúvida, é uma estratégia de aproximação dos estudantes universitários aos desafios da construção técnica, social e política do SUS ${ }^{(2,4)}$.

\section{OBJETIVO}

Conhecer o significado do VER-SUS para a formação do acadêmico da área da saúde, com vistas a contribuir 
com os debates sobre a inclusão curricular do SUS nos projetos político-pedagógicos de cursos de graduação.

\section{MÉTODOS}

É um estudo exploratório qualitativo e o projeto de pesquisa foi aprovado pelo Comitê de Ética em Pesquisa da Universidade Federal de São Paulo, Processo n.o 1613/04.

\section{A coleta dos dados e os sujeitos do estudo}

Os dados foram coletados por meio de um instrumento com questões semi-estruturadas direcionadas para captar atitudes, valores e crenças dos graduandos durante a experiência do VER-SUS. O instrumento foi enviando aos sujeitos do estudo via correio (eletrônico e/ou postal), após um primeiro contato dos pesquisadores com os estudantes de graduação.

Para operacionalizar a coleta de dados e definição dos sujeitos, utilizamos a seguinte estratégia. Fomos aos encontros estudantis que estavam ocorrendo nos meses de março e abril de 2005 no Estado de São Paulo e explicávamos aos estudantes que participaram do VERSUS, o projeto de pesquisa que estávamos realizando. Assim, conseguimos a adesão de alguns ao estudo, para os quais, imediatamente entregávamos o Termo de Consentimento Livre e Esclarecido, informando que o instrumento para coleta de dados seguiria via correio eletrônico, e que após o preenchimento deveriam nos enviar os documentos pelo correio postal.

Paralelo a esta estratégia, entramos em contato via correio eletrônico com os estudantes que participaram do VER-SUS e não estiveram presentes nessas reuniões do movimento estudantil. Explicamos o nosso interesse, e àqueles que concordaram em participar enviamos o Termo de Consentimento Livre e Esclarecido e o instrumento para coleta de dados. As respostas vieram via correio eletrônico e os termos de consentimento pelo correio postal.

Desta forma entramos em contato com 30 estudantes, dos quais dez responderam no prazo estipulado. Partindo do pressuposto teórico que a pesquisa qualitativa não requer determinação amostral para a definição dos participantes, e que a seleção dos sujeitos resulta de uma avaliação da representatividade social (não estatística) das pessoas ${ }^{(5)}$, iniciamos a análise dos dados com os que atenderam nossa solicitação no tempo estabelecido.

\section{Análise dos dados}

Os dados foram analisados por meio da técnica da análise temática, tomando como base os temas que emergiram dos relatos dos sujeitos e representaram unidades de significação capazes de denotar os valores de referência e os modelos de comportamento presentes nas narrativas ${ }^{()}$.
Para a realização da análise dos dados empíricos partimos da premissa de que os estudantes participantes da pesquisa compartilhavam a experiência de já terem vivenciado o VERSUS, portanto possuíam amplas condições para objetivarem o nosso objeto de estudo. Tal constatação nos levou a realizar recortes temáticos nas narrativas; e foram três os fios condutores desse processo de análise: o conhecimento sobre o SUS no processo de formação do graduando, o VERSUS como instrumento para inserção na política estudantil e/ou em outros movimentos sociais; e o VER-SUS e a formação acadêmica.

A análise foi centrada nos dados coletados. Para assegurar o sigilo acordado com os sujeitos da pesquisa mantivemos a distinção entre os entrevistados e as entrevistadas colocando $\mathrm{M}$ ou $\mathrm{F}$ para caracterizar respectivamente o sexo masculino ou feminino; a idade em anos e, o curso que estava fazendo.

\section{RESULTADOS}

Fizeram parte deste estudo dez graduandos da área da saúde, sendo seis mulheres e quatro homens, distribuídos na faixa etária de 20 a 38 anos, oriundos de instituições de ensino públicas e privadas; dos cursos de enfermagem, fisioterapia, medicina, odontologia e psicologia, que participaram do VER-SUS no Estado de São Paulo nos meses de agosto e setembro de 2004.

\section{O conhecimento sobre o SUS no processo de formação do graduando}

O primeiro aspecto considerado na coleta de dados foi a necessidade de conhecer a visão dos estudantes em relação ao SUS antes da vivência.

Conbecia a parte assistencial do SUS. Via inúmeros problemas, mas por desconhecer o sistema em todos os seus segmentos, não encontrava, com clareza, as causas. Não conhecia os eixos de gestão e controle social. (F, 23, enfermagem)

Não tinha conhecimento, nenbum conbecimento sobre a estrutura e funcionamento do sistema. Minha visão era de um sistema falido, de hospitais cheios de fila (...). Enfim, uma visão totalmente negativa e desinformada. (F, 22, psicologia)

Minha visão era de um sistema totalmente decadente e sem futuro, não sabia como era a realidade e muito menos como funcionava (...). (F, 20, odontologia)

Por meio dessas narrativas observamos que alguns estudantes desconheciam ou tinham uma visão limitada do SUS no que concerne a seu funcionamento. Esse contexto corrobora com a premissa de que a formação dos recursos humanos para o setor é um dos grandes desafios do sistema.

Outros retrataram o desconhecimento do funcionamento do sistema - principalmente no que tange ao controle social - quando expressaram o seu descontentamento enquanto 
usuários dos serviços oferecidos pelo SUS.

(...) me decepcionei pela demora inicialmente, depois pelo atendimento e assim seguia várias outras decepções com o sistema. (F, 25, fisioterapia)

Mas das vezes que eu usei, (...), a impressão não foi boa: filas demoradas, erros nos serviços que causavam uma demora ainda maior (...). (M, 20, fisioterapia).

Todavia outros relataram conhecer alguns aspectos do SUS.

(...) cheguei ao estágio do VER-SUS com uma bagagem teórica interessante (...). Tive a oportunidade de estudar os princípios e diretrizes do SUS, sem, contudo, ter a capacidade de dimensionar a importância e o impacto na realidade de cada um. (F, 21, enfermagem)

A minha visão já contemplava muitas questões e muitas concepcões a respeito do SUS, porém de forma bastante restritiva. (M, 26, enfermagem)

As narrativas mostram que alguns estudantes já possuíam alguma concepção teórica sobre o sistema, porém tinham um conhecimento restrito sobre sua aplicabilidade na prática.

\section{O VER-SUS como instrumento para inserção na política estudantil e/ou em outros movimentos sociais}

Este tema emergiu da coleta dados a respeito da influência do VER-SUS na vida estudantil dos sujeitos da presente pesquisa.

Comecei a participar ativamente do movimento estudantil, ou seja, minha vida estudantil não se limita à sala de aula e vai além dos livros. (F, 20, odontologia)

Os conhecimentos adquiridos durante a vivência me fižram refletir sobre as disciplinas que curso e sobre a bibliografia que usamos, além dos estágios. Além disso, passei a participar de coletivos estudantis e sociais. (F, 22, psicologia)

Observamos o desejo dos estudantes de atuarem em movimentos sociais. A vivência os estimulou a crer na possibilidade de mudanças começando na própria universidade. $\mathrm{O}$ movimento estudantil organizado redigiu um manifesto de apoio* ao SUS, com a intenção de sensibilizar as pessoas, principalmente aquelas

\footnotetext{
* Manifesto de Apoio. Os estudantes da área da saúde pelo SUS e em defesa da vida. Assinado pelos: Conselho Nacional dos Estudantes de Psicologia, Direção Executiva Nacional dos Estudantes de Fonoaudiologia, Direção Executiva Nacional dos Estudantes de Medicina, Executiva Nacional dos Estudantes de Enfermagem, Executiva Nacional dos Estudantes de Fisioterapia, Executiva Nacional dos Estudantes de Nutrição, Executiva Nacional dos Estudantes de Farmácia, Executiva Nacional dos Estudantes de Biomedicina, Pró-executiva Nacional dos Estudantes de Terapia Ocupacional, Pró-executiva Nacional dos Estudantes de Odontologia. Brasília (DF). 2004. (mimeo)
}

relacionadas à saúde, para que lutem em sua defesa, pois além de serem usuários, colaboram para a implementação do sistema. Acreditam que, com a mobilização, as conquistas podem ser mais efetivas.

Tendo em vista este processo, as descrições de alguns estudantes que participaram do VER-SUS corroboram com a idéia de que atores sociais se agrupam em movimentos em busca de uma mesma conquista.

Na vida estudantil, trouxe a necessidade de aproximar o meio acadêmico em geral à realidade da saúde pública. No entanto, isso muitas vezesé complicado, pois uma pessoa só, às vezes não consegue induz̨ir essa transformação. ( $M, 23$, medicina)

(...) existe uma grande parcela do SUS que ainda precisa ser construida e que depende de cada profissional envolvido na rede (...). Para tanto, é necessário que saibamos, desde a faculdade, qual é o nosso papel e o que podemos fazer para sermos atuantes como atores sociais que somos. (F, 38, enfermagem)

O VER-SUS, entre outros papéis, estimula a organização social e política dos estudantes. Tal constatação nos remete ao pensamento de que o indivíduo se organiza quando percebe que sua ação individual se identifica com a de outros indivíduos.

\section{O VER-SUS e a formação acadêmica}

Outro tema que emergiu das narrativas leva-nos a refletir sobre uma das finalidades deste estudo, ou seja, fomentar discussões acerca das mudanças políticopedagógicas na graduação para a capacitação de profissionais para o SUS.

(...) participei da mudança de currículo no último período da graduação inserindo importantes discussões e espaço de atuação a respeito do SUS. (M, 26, enfermagem)

(...) esta influência pode despertar um senso crítico e reação/ participação do aluno, seja como usuário, seja como profissional. (F, 25, fisioterapia)

A vivencia ampliaria os conbecimentos dos graduandos, os tornaria mais críticos, mais responsáveis, daria mais motivação para estudar, aprender e ensinar. (F, 20, odontologia)

Para alguns estudantes, a vivência é uma alternativa adequada e capaz de angariar resultados satisfatórios na formação para o SUS, por meio das críticas e discussões a respeito, ainda na graduação. Esta visão traria contentamento e satisfação para o graduando estimulando-o a ser mais participativo nas decisões educacionais de sua universidade.

Como apenas dois acadêmicos de minha instituição puderam fazer parte do VER-SUS, é dificil a influência que podemos ter, mas acredito que com mais pessoas fazendo parte deste estágio, pode ficar mais fácil. ( $M, 20$, enfermagem) 
Mas isso não é o suficiente, porque sempre seria algo pontual que poucos estudantes teriam acesso (...). Para haver uma mudança efetiva na graduação, os próprios professores precisariam passar por experiências parecidas e terem vontade de realmente fazer diferente. (M, 20, fisioterapia)

A troca entre professores e alunos que participaram da vivência pode auxiliar na reestruturação do currículo, pensar os estágios no SUS de forma integral, ou seja, possibilitar ao aluno uma visão completa do sistema (...). (F, 22, psicologia)

A participação dos professores junto aos graduandos na vivência poderia efetivar uma mudança mais concreta na graduação e não apenas algo localizado, no qual o estudante participa, mas a universidade minimiza o valor da experiência.

Se a universidade aglomera as cabecas pensantes, os intelectuais da sociedade, ela tem papel ativo direto na transformação da sociedade, responsabilidade que hoje está sendo muito pouco cumprida na maioria das instituiçoes. (M, 20, fisioterapia)

\section{DISCUSSÃO}

Para nós, a questão inerente à formação de estudantes da área da saúde não se esgota na discussão de políticas governamentais focais - no caso o VER-SUS. Engloba, também, uma ampla discussão sobre a política pedagógica usada para o ensino nas universidades, pautada quase sempre na abordagem pedagógica tradicional, conservadora, em que a relação professor-estudante é vertical. $\mathrm{O}$ papel do professor está intimamente ligado à transmissão de certo conteúdo que é pré-definido e que constitui o próprio fim da existência escolar; o estudante por sua vez é submetido a absorver o conhecimento que o professor detém, idealizando a concepção de que apenas esse tem o potencial para transmitir conhecimento ${ }^{(7)}$.

A questão da formação de profissionais para o SUS, nos últimos anos, passa a ocupar um lugar central, finalístico às políticas de saúde; torna inconcebível o não investimento na área da educação - seja a voltada aos graduandos e pós-graduandos, seja a direcionada aos trabalhadores já inseridos no sistema, por meio do exercício da educação permanente em saúde ${ }^{(8)}$.

Considerando que os currículos representam a perspectiva filosófica de um grupo (seus valores, suas atitudes e suas crenças), o grande desafio na formação de estudantes na área da saúde para atuarem no SUS, segundo o nosso prisma, encontra-se na tomada de consciência, por parte de segmentos docentes, sobre a premência de se instrumentalizar técnica, científica e politicamente os futuros profissionais, frente às questões sociais e políticas do país - em particular as relacionadas ao setor saúde ${ }^{(9)}$.

Cremos que experiências como o VER-SUS podem desencadear o processo de mudança de mentalidade nos atores sociais das universidades, com conseqüente mudança de atitude profissional, o que nos parece fundamental, já que a elaboração do saber sobre o SUS produz alteração nas relações e práticas de poder entre os defensores da saúde enquanto direito e aqueles que crêem que é mercadoria.

Assim, a formação de profissionais competentes para atuar no SUS implica em um processo de revisão paradigmática, pautado na Ética, no compromisso com a saúde enquanto direito de todos e dever do Estado. As universidades com os cursos voltados à área da saúde precisam tomar uma posição. Das públicas, mais do que nunca se espera a maximização de esforços para a formação de futuros profissionais críticos e competentes para atuar no SUS, tornando indissociável a relação entre a teoria e a prática, fazendo imprescindível a revisão dos locais utilizados para o ensino.

O VER-SUS é um modelo possível para contribuir com esse processo formativo. Não é o único, mas incorpora questões atuais sobre a formação de recursos humanos para o SUS. É neste contexto que trazemos à discussão o significado do VER-SUS na formação dos estudantes com vistas a subsidiar o diálogo com as universidades sobre a formação de graduandos para o SUS.

Os resultados deste estudo revelam que a participação no VER-SUS contribuiu para o aprimoramento individual dos estudantes sobre o SUS, destacando a sua importância enquanto política pública. Os graduandos relataram que essa vivência minimizou o preconceito que tinham sobre esse Sistema. Mencionaram, também, que a experiência foi um fator desencadeador da participação em movimentos sociais, particularmente no movimento estudantil. Destacaram, ainda, que apesar da qualidade da vivência, ela é de fórum individual, dificilmente aplicável no coletivo da academia, em decorrência de pouco conhecimento dos docentes sobre o SUS, bem como reduzido interesse dos estudantes - fatores que apontam como passíveis de intervenção por meio de capacitação e motivação institucional.

Parte dos graduandos da área da saúde não é formada para compreender o SUS, não obstante, percebemos que o desconhecimento alia a uma visão negativista e sem futuro. Muitos usam o sistema para seu aprendizado técnicocientífico, no entanto, não são formados para atuar nele de forma crítica, reflexiva. O que nos remete à reflexão sobre a responsabilidade dos professores e pesquisadores universitários, na qual a despreocupação com as finalidades sociais do conhecimento gerado se constitui, muitas vezes, em fator impeditivo para efetivação do SUS.

Nesta perspectiva, torna-se imprescindível a efetivação de políticas públicas que integrem as áreas da Saúde e da educação, garantindo que as práticas educativas realizadas no SUS configurem dispositivos para a análise e, quiçá intervenção conjunta, das experiências de saúde locais; que possibilitem o diálogo entre o aparelho formador e os serviços de saúde, 
fortalecendo a participação popular e valorizando os saberes locais ${ }^{(8)}$. Tal ação implica na mudança de mentalidade, tanto dos gestores quanto dos trabalhadores da saúde e da educação, bem como do movimento social organizado por meio dos conselhos populares e/ou conselhos gestores de saúde. A integração entre esses diferentes segmentos é necessária no processo de formação para a assistência à saúde ${ }^{(10)}$.

Quanto aos graduandos, não temos dúvida da necessidade de viverem a experiência sobre e no SUS, para que quando profissionais possam compreendê-lo e, assim, colaborar para seu desenvolvimento. É preciso ter em mente que a formação na área da saúde é, antes de tudo, um instrumento para transformações no setor saúde. Porém, a universidade precisa entender que faz parte do SUS e, de forma dialética e dialógica, compreender que ao mesmo tempo em que contribui com a formação de profissionais para o SUS, busca no sistema subsídios para questionar a sua própria práxis.

Tal atitude corrobora com outros movimentos voltados à formação de recursos humanos mais críticos e comprometidos com sistemas de saúde capazes de assegurar o acesso universal da população aos serviços qualificados de saúde. Destacamos a VII Reunião Regional dos Observatórios de Recursos Humanos em Saúde, realizada em Toronto em 2005, e no âmbito brasileiro, por meio de parceria entre os Ministérios da Saúde e da Educação, o Programa Nacional de Reorientação da Formação Profissional em Saúde - Pró-Saúde ${ }^{(11-12)}$.

\section{CONSIDERAÇÕES FINAIS}

O significado do VER-SUS para os estudantes vêm carregado - não só da experiência vivenciada, mas, sobretudo pelos valores, crenças e atitudes que dão sentido ao imaginário de cada um. A defesa do SUS, portanto, suscita o diálogo entre o potencial humano que o estudante tem, o conteúdo teórico-prático que lhe é oferecido na graduação e a sua participação em movimentos sociais organizados - entre eles, o estudantil.

Entendemos ser imprescindível a efetiva cooperação entre os Ministérios da Educação e da Saúde para que se viabilize a integração entre a universidade e o SUS. Para tanto, são necessários acordos e parcerias em todo o território nacional, envolvendo movimentos sociais progressistas, que além de reivindicarem melhorias em diversos setores - educação, saúde, trabalho, moradias - sejam capazes de defender o SUS, como usuários e/ ou como trabalhadores do sistema.

Nesta perspectiva, vemos o movimento estudantil como mais um parceiro e, sobretudo, como o lócus de formação política dos futuros profissionais. Inserido na formulação, execução e avaliação de políticas pode contribuir para a construção de mudanças positivas na saúde e educação.

Desta forma, acreditamos que vivências como a propiciada pelo VER-SUS contribuem, mesmo que de forma focal, para a formação técnica, científica e política dos graduandos da área da saúde, na perspectiva interdisciplinar e intersetorial. Como tal, deve ser preservada e ampliada.

\section{AGRADECIMENTOS}

Agradecemos ao Gerson Calsolari Catallani a participação no processo de coleta de dados.

\section{REFERÊNCIAS}

1. Brasil. Ministério da Saúde. Secretaria Executiva. Sistema Único de Saúde (SUS): princípios e conquistas. Brasília: Ministério da Saúde; 2001.

2. Brasil. Ministério da Saúde. Secretaria de Gestão do Trabalho e da Educação na Saúde. Departamento de Gestão de Educação na Saúde. VER-SUS Brasil: Caderno de Textos. Brasília: Ministério da Saúde; 2004.

3. Silva KL, Sena RR. Nursing education: seeking criticalreflexive education and professional competencies. Rev Latinoam Enferm. 2006; 14(5): 755-61.

4. Brasil. Ministério da Saúde. Secretaria de Gestão do Trabalho e da Educação na Saúde. Departamento de Gestão da Educação na Saúde. Política de educação e desenvolvimento para o SUS: caminhos para a educação permanente em saúde: pólos de educação permanente em saúde. Brasília: Ministério da Saúde; 2004.

5. Thiollent MJM. Crítica metodológica, investigação social e enquete operária. 5a ed. São Paulo: Polis; 1987.

6. Minayo MCS. O desafio do conhecimento: pesquisa

qualitativa em saúde. 10a. ed. São Paulo: Hucitec; 2007.

7. Freire P. Pedagogia do oprimido. 12a ed. Rio de Janeiro: Paz e Terra; 1983.

8. Ceccim RB. Educação permanente em saúde: desafio ambicioso e necessário. Interface Comun Saude Educ. 2005; 9(16): 161-77.

9. Brêtas ACP. As enfermeiras, o poder, a história: um estudo exploratório sobre mentalidades [dissertação]. Campinas: Faculdade de Educação da Universidade Estadual de Campinas; 1994.

10. Ceccim RB, Feuerwerker LCM. Mudança na graduação das profissões de saúde sob o eixo da integralidade. Cad Saude Publica = Rep Public Health. 2004; 20(5): 1400-10.

11. Mendes IAC, Marziale MHP. Década de recursos humanos em saúde: 2006-2015. Rev Latinoam Enferm. 2006;14(1):12.

12. Mendes IAC, Marziale MHP. Sistemas de saúde em busca de excelência: os recursos humanos em foco. Rev Latinoam Enferm. 2006; 14(3): 303-7. 